Iternational Electronic Journal of Algebra

VOLUME 27 (2020) 102-113

DOI: $10.24330 /$ ieja.662972

\title{
ON S-EXTENDING MODULES
}

M. E. Tabarak, M. A. Kamal, M. H. Elbaroudy and S. E. D. S. Hussien

Received: 30 January 2019; Revised: 20 August 2019; Accepted: 24 August 2019

Communicated by Abdullah Harmancı

\begin{abstract}
Here we introduce and study the concept of relative superfluous injectivity, which is a generalization of relative injectivity. We show some of the properties that hold true for relative injectivity still hold for relative superfluous injectivity. We also introduce and characterize the new concept of superfluous extending modules. Finally, we make use of relative superfluous injectivity to study direct sums of superfluous extending modules.
\end{abstract}

Mathematics Subject Classification (2010): 16D50, 16D70, 16D80

keywords: Superfluous injective module, large submodule, superfluous extending module

\section{Introduction}

Throughout all modules are unital right $R$-modules over an arbitrary associative ring $R$ with unity. The generalization of relative injectivity for module was studied by many authors, and this has been by adding extra condition on the homomorphism in the defining diagram (cf. E. Mermut, C. Santa-Clara and P. F. Smith [6], H. Q. Dinh [3] and M. F. Yousif and Y. Zhou [8]). Here we introduce the concept of $N$ being $M$-superfluous-injective, which is a generalization of $N$ being $M$-injective for modules (Definition 2.3). We define in Definition 2.10, Definition 2.11 what is meant by $C o n-S$-complement and $C o n$ - $S$-closed submodule of a module. In Theorem 2.16 we give an equivalent condition of the diagram in Definition 2.3 by a property on a decomposition form, in fact we prove that $M_{2}$ is $M_{1}$-superfluousinjective if and only if $M=C \oplus M_{2}$ holds for every $C o n-S$-complement $C$ of $M_{2}$ in $M$, whenever $M=M_{1} \oplus M_{2} ; M_{1}$ has the condition $\left(S_{*}\right)$. This is analogue with the known fact about relative injectivity [2]. In Section 3, we introduce a generalization of extending modules. This has been done by relaxing the defining condition of such modules. In fact we concentrate on $C o n$ - $S$-closed submodules, and examine for being direct summands. We show in the case of $S$-extending modules in contrary of extending modules, that the class of summands does not coincide with the class of Con-S-closed submodules. In Remark 3.3, we mention when such classes coincide. 
In [4], it was shown that a direct sum of extending modules $M_{1}$ and $M_{2}$ is extending if and only if every closed submodule with zero intersection with $M_{1}$ or with $M_{2}$ is a direct summand (Lemma 7.9). Here in Section 4, we prove that a direct sum of $S$-extending modules $M_{1}$ and $M_{2}$, with $0 \neq \operatorname{Rad}(C) \unlhd C$, for every proper $C o n$ - $S$-closed submodule $C$ of $M$, with $C \cap M_{i} \neq 0, i=1,2$ is $S$-extending if and only if every Con-S-closed submodule with zero intersection with $M_{1}$ or with $M_{2}$ is a direct summand and also we prove that a direct sum of $S$-extending modules $M_{1}$ and $M_{2}$, with $S I C$-property, is $S$-extending if and only if every Con-S-closed submodule with zero intersection with $M_{1}$ or with $M_{2}$ is a direct summand. Finally in Theorem 4.9 and Theorem 4.10, we make use relatively superfluous injectivity to prove that a finite direct sum of $S$-extending modules is $S$-extending. We use $L \leq M, N \ll M$ and $K \unlhd M$ to denote that $L$ is a submodule, $N$ is a superfluous submodule, and $K$ is a large submodule of $M$, respectively, for more details see [1].

\section{Relative superfluous injective modules}

In this section we study and analyze the properties of the concept of superfluous injectivity.

Definition 2.1. Let $N$ be a submodule of a module $M . N$ is called a Con-Ssubmodule in $M$ relative to $L$ (denoted by Con- $S_{L}$-submodule in $M$ ) if there exists a non-zero superfluous submodule $K$ of $L$, with $K \leq N$. If $L=M$, then we just say that $N$ is a $C o n-S$-submodule of $M$.

Definition 2.2. A monomorphism $f: N \rightarrow M$ is called a $C o n-S$-monomorphism if $f(N)$ is a $C o n-S$-submodule of $M$.

Definition 2.3. Let $M$ be an $R$-module. A module $N$ is said to be $M$-superfluousinjective if for every Con-S-monomorphism $f: L \rightarrow M$, any homomorphism $\varphi: L \rightarrow N$, there exists a homomorphism $\psi: M \rightarrow N$ such that $\psi f=\varphi$.

Lemma 2.4. The following are equivalent for modules $N$ and $M$ :

(1) $N$ is $M$-superfluous-injective,

(2) For every Con-S-submodule $L$ of $M$, any homomorphism $\varphi: L \rightarrow N$ can be extended to a homomorphism $\psi: M \rightarrow N$.

Proof. (2) $\Rightarrow(1)$ : Let $f: L \rightarrow M$ be a Con-S-monomorphism, and $\varphi: L \rightarrow N$ be a homomorphism. It follows that $f(L)$ is a Con-S-submodule of $M$. Hence, by (2), there is a homomorphism $\psi: M \rightarrow N$ such that $\psi$ extends $\left.\varphi f^{-1}\right|_{f(L)}$. Therefore $\psi f=\varphi$; and hence we have (1).

$(1) \Rightarrow(2)$ : It is clear. 
Remark 2.5. (a) Let $M$ be an $R$-module, with no non-trivial superfluous submodules. Then every $R$-module $N$ is $M$-superfluous-injective module. For examples:

(1) Every $\mathbb{Z}$-module $N$ is a $\mathbb{Z}$-superfluous-injective module.

(2) If $M$ is a semisimple $R$-module, then every $R$-module $N$ is $M$-superfluousinjective module.

(3) If $M$ is a semiprimitive $R$-module, then every $R$-module $N$ is $M$-superfluousinjective module.

(b) Let $M$ be an $R$-module, with $0 \neq \operatorname{Rad}(M) \unlhd M$. Then $M$-superfluousinjectivity and $M$-injectivity are equivalent.

The examples, which are given above, show that there are many $M$-superfluousinjective, which are not $M$-injective modules.

Proposition 2.6. Let $N$ and $M$ be R-modules. Then the following are equivalent:

(1) $N$ is $M$-superfluous-injective.

(2) $N$ is $M_{1}$-superfluous-injective, for each submodule $M_{1}$ of $M$.

(3) $N$ is $M / L$-superfluous-injective, for each superfluous submodule $L$ of $M$.

Proof. (1) $\Rightarrow(2)$ : Let $M_{1}$ be a submodule of $M$, and $\varphi: L \rightarrow N$ a homomorphism, for some Con-S-submodule $L$ of $M_{1}$. It is easy to show that $L$ is Con-Ssubmodule of $M$, and hence, by (1), $\varphi: L \rightarrow N$ can be extended to a homomorphism $\psi: M \rightarrow N$. Therefore $\psi$ restricted to $M_{1}$ extends $\varphi$.

(2) $\Rightarrow(3)$ : Let $L$ be a superfluous submodule of $M$. We show that $N$ is $M / L$ superfluous-injective. To this end, let $\varphi: M_{1} / L \rightarrow N$ be a homomorphism, for some $C o n-S$-submodule $M_{1} / L$ of $M / L$. Hence $M_{1} / L$ contains a nonzero superfluous submodule $K / L$ in $M / L$. It is clear that $\mathrm{K}$ is superfluous in $M$; and hence $M_{1}$ is a $C o n-S$-submodule of $M$. Let $\pi: M \rightarrow M / L$ be the natural epimorphism. As $N$ is $M$-superfluous-injective, we have that $\left.\varphi \pi\right|_{M_{1}}$ can be extended to a homomorphism $\psi: M \rightarrow N$; i.e. $\psi\left(m_{1}\right)=\varphi\left(m_{1}+L\right)$, for all $m_{1} \in M_{1}$. Since $\psi(l)=0$, for all $l \in L$, we have that $\psi: M \rightarrow N$ defines a homomorphism $\alpha: M / L \rightarrow N$ by $\alpha(m+L)=\psi(m)$, for all $m+L \in M / L$. It is easy to show that $\alpha: M / L \rightarrow N$ extends $\varphi: M_{1} / L \rightarrow N$.

(3) $\Rightarrow(1)$ : It is clear by taking $L=0$.

Proposition 2.7. Let $N$ and $M$ be R-modules. Then the following are equivalent:

(1) $N$ is $M$-superfluous-injective.

(2) $L$ is $M$-superfluous-injective, for each direct summand $L$ of $N$.

(3) $N^{\prime}$ is $M$-superfluous-injective, for each isomorphic copy $N^{\prime}$ of $N$.

(4) $N$ is $M^{\prime}$-superfluous-injective, for each isomorphic copy $M^{\prime}$ of $M$. 
Proof. (1) $\Rightarrow(2)$ : Let $N=L \oplus L^{\prime}$, and $\varphi: M_{1} \rightarrow L$ be a homomorphism, for some $C$ on- $S$-submodule $M_{1}$ of $M$. As $N$ is $M$-superfluous-injective module, there is an extension homomorphism $\psi: M \rightarrow N$ of $\varphi$. It follows that $\pi_{L} \psi$ extends $\varphi$, where $\pi_{L}: N \rightarrow L$ is the projection of $N$ onto $L$.

(2) $\Rightarrow(1)$ : It is clear by taking $L=N$.

$(1) \Leftrightarrow(3)$ : The proof is clear.

$(1) \Rightarrow(4)$ : Let $L$ be a $C o n-S$-submodule of $M^{\prime}$, then there exists a superfluous submodule $K$ of $M^{\prime}$ in $L$ and let $\varphi: L \rightarrow N$ be a homomorphism and $f: M^{\prime} \rightarrow M$ ba an isomorphism. Thus $f(K) \ll M$ and $f(K) \leq f(L)$. Then $f(L)$ is a Con-Ssubmodule of $M$. Since $N$ is $M$-superfluous-injective, then there exists $g: M \rightarrow N$ such that $g(x)=\varphi f^{-1}(x)$, for all $x \in f(L)$. Therefore $g f(l)=\varphi f^{-1}(f(l))=\varphi(l)$ for all $l \in L$. Thus $g f$ extends $\varphi$.

By the similar way we can prove that $(4) \Rightarrow(1)$.

Proposition 2.8. Let $M$ be an $R$-module and $\left\{N_{i}: i \in I\right\}$ be a family of $R$ modules. Then $\Pi_{i \in I} N_{i}$ is $M$-superfluous-injective if and only if $N_{i}$ is $M$-superfluousinjective, for each $i \in I$.

Proof. It is clear.

Corollary 2.9. Let $M$ be an $R$-module and $\left\{N_{i}: i=1, \ldots, n\right\}$ be a family of $R$ modules. Then $\oplus_{i=1}^{n} N_{i}$ is $M$-superfluous-injective if $N_{i}$ is $M$-superfluous-injective, for each $i=1, \ldots, n$.

Definition 2.10. A submodule $L$ of a module $M$ is called a $C o n-S$-complement of a submodule $K$ in $M$, if it is a $C o n-S$-submodule of $M$, and maximal with respect to $L \cap K=0$.

Definition 2.11. A submodule $L$ of a module $M$ is called a $C o n-S$-closed submodule of $M$, if it is a $C o n-S$-submodule of $M$, and has no proper large extension submodules of $M$.

Remark 2.12. Let $M$ be a module, we have the following:

(1) Every maximal large extension of a $C o n-S$-submodule of a module $M$ is Con-S-closed in $M$.

(2) Let $N$ be a $C o n-S$-submodule, and $K$ be a submodule of a module $M$; with $N \cap K=0$. Then $N$ is a Con-S-complement of $K$ if and only if $N$ is Con-S-closed, and $N \oplus K$ is large in $M$.

Definition 2.13. A module $M$ is said to have the condition $\left(S_{*}\right)$, if for every superfluous submodule $K$ of $M$ and $K \leq N \unlhd M$, we have that $K \ll N$. 
Example 2.14. Any semiprimitive module and any semisimple module has $\left(S_{*}\right)$.

Lemma 2.15. Let $M=M_{1} \oplus M_{2}$ be a module where $M_{1}$ has $\left(S_{*}\right), N$ be a Con$S$-submodule of $M_{1}$, and $f: N \rightarrow M_{2}$ be a homomorphism. Let $N \leq X \leq M_{1}$, and $\varphi: X \rightarrow M_{2}$ be a maximal extension of $f$. Then the submodule $X^{*}=\{x+\varphi(x):$ $x \in X\}$ of $M$ is a Con-S-complement of $M_{2}$ in $M$.

Proof. It is clear that $X^{*} \cap M_{2}=0$. Let $\pi_{i}: M \rightarrow M_{i}, i=1,2$, be the natural projections. Since $N$ is a Con-S-submodule of $M_{1}$, then there exists $0 \neq K \leq N$, with $K \ll M_{1}$. Hence $K^{*}=\{k+\varphi(k): k \in K\} \leq \pi_{1}\left(K^{*}\right)+\pi_{2}\left(K^{*}\right)=K+\varphi(K)$. Since $K \ll M_{1}, K \leq N \leq X \unlhd M_{1}$ and $M_{1}$ has $\left(S_{*}\right)$, then $K \ll X$ and since homomorphic images of superfluous submodules are superfluous, then $\varphi(K) \ll M_{2}$ and so, $\varphi(K) \ll M$. As finite sums of superfluous submodules are superfluous, thus $K+\varphi(K) \ll M$, hence $K^{*} \ll M$ and $K^{*} \leq X^{*}$; i.e. $X^{*}$ is a $C o n-S$-submodule of $M$. Now let $X^{*} \leq D$, with $D \cap M_{2}=0$. Clearly $\left.\pi_{1}\right|_{D}$ is monomorphism and $X=\pi_{1}\left(X^{*}\right) \leq \pi_{1}(D)$, define $\psi: \pi_{1}(D) \rightarrow M_{2}$ by $\psi\left(\pi_{1}(d)\right)=\pi_{2}(d)$ for all $d \in D$. Then $\psi$ extends $\varphi: X \rightarrow N$. Thus $X=\pi_{1}(D)$ by maximality of $\varphi$, and hence $X^{*}=D$. Which shows that $X^{*}$ is a Con-S-complement of $M_{2}$.

Theorem 2.16. Let $M=M_{1} \oplus M_{2}$ where $M_{1}$ has $\left(S_{*}\right)$. Then the following are equivalent:

(1) $M_{2}$ is $M_{1}$-superfluous-injective.

(2) If $N$ is a Con-S-submodule of $M$, with $N \cap M_{2}=0$, then $M=C \oplus M_{2}$, for some submodule $C$ containing $N$.

(3) $M=C \oplus M_{2}$, for each Con-S-complement $C$ of $M_{2}$ in $M$.

Proof. (1) $\Rightarrow(2)$ : Let $N$ be a Con-S-submodule of $M$ with $N \cap M_{2}=0$. As $N$ is a $C o n-S$-submodule of $M$; i.e. $N$ contains a non-zero submodule $K$ superfluous in $M$, it follows that $\pi_{1}(K)$ is superfluous in $M_{1}$, where $\pi_{1}: M \rightarrow M_{1}$ is the projection onto $M_{1}$. Hence by (1), there exists a homomorphism $\varphi: M_{1} \rightarrow M_{2}$ such that $\left.\varphi \pi_{1}\right|_{N}=\left.\pi_{2}\right|_{N}$, where $\pi_{2}: M \rightarrow M_{2}$ is the projection onto $M_{2}$. Hence, for all $n \in N$, we have $n=\pi_{1}(n)+\pi_{2}(n)=\pi_{1}(n)+\varphi \pi_{1}(n) \in M_{1}^{*}=\left\{m_{1}+\varphi\left(m_{1}\right)\right.$ : $\left.m_{1} \in M_{1}\right\}$, where $M=M_{1}^{*} \oplus M_{2}$.

$(2) \Rightarrow(3)$ : Let $C$ be a $C o n-S$-complement of $M_{2}$ in $M$. Then $C \cap M_{2}=0$, so by (2) there exists $L \geq C$ such that $M=L \oplus M_{2}$. Hence $C=L$ (due to $L \cap M_{2}=0$ ). $(3) \Rightarrow(1)$ : Let $N$ be a Con-S-submodule of $M_{1}$, and $f: N \rightarrow M_{2}$ be a homomorphism. Let $\varphi: N \leq X \rightarrow M_{2}$ be a maximal extension of $f$. By Lemma 2.15, we have that $X^{*}=\{x+\varphi(x): x \in X\}$ is a Con-S-complement of $M_{2}$. Hence, 
by (3), $M=X^{*} \oplus M_{2}$. It is easy to show that $X=M_{1}$, and therefore $M_{2}$ is $M_{1}$-superfluous-injective.

\section{Superfluous extending modules}

In this section we study simultaneously the dual concepts of superfluousness and largeness on modules, namely the condition that each $C o n-S$-submodule of a module $M$ is large in a direct summand of $M$. Such modules will be called $S$-extending modules.

Definition 3.1. A module $M$ is called a superfluous extending (for short $S$ extending) module, if every $C o n-S$-submodule of $M$ is large in a direct summand of $M$.

Theorem 3.2. The following are equivalent for a module $M$ :

(1) $M$ is $S$-extending.

(2) Every $S$-closed submodule of $M$ is a direct summand.

(3) For every Con-S-submodule $A$ of $M$, there exists a decomposition $M=M_{1} \oplus M_{2}$ such that $M_{1}$ contains $A$, and $A \oplus M_{2}$ is large in $M$.

(4) If $A$ is a closed submodule of $M$ and $B$ a direct summand of $M$; with $A \cap B$ Con-S-closed submodule in $B$, then $A \cap B$ is a direct summand of $M$.

Proof. $(1) \Rightarrow(2)$ : Follows from definition of $C o n-S$-closed submodule.

$(2) \Rightarrow(3)$ : Let $A$ be a $C o n-S$-submodule of $M$, it follows from Remark 2.12 that a maximal large extension $C$ of $A$ in $M$ is $C o n$ - $S$-closed. Hence, by (2), $C$ is a direct summand of $M$; and therefore $M=C \oplus M_{2}$, with $A \oplus M_{2}$ is large in $M$, for some submodule $M_{2}$ of $M$.

$(3) \Rightarrow(4)$ : Let $A$ be a closed submodule of $M$, and $B$ be a direct summand of $M$; with $A \cap B C$ on- $S$-closed submodule of $B$, then $A \cap B$ is a $C o n$ - $S$-closed submodule of $M$. To avoid triviality, we may consider $A, B \neq M$. Now, by (3), there is a decomposition $M=M_{1} \oplus M_{2}$ such that $M_{1}$ contains $A \cap B$, and $A \cap B \oplus M_{2}$ is large in $M$. As $A \cap B$ is a closed submodule in $M$, we have that $A \cap B=M_{1}$.

$(4) \Rightarrow(1)$ : Let $A$ be a $C o n-S$-submodule of $M$. Hence, from Remark 2.12, a maximal large extension of $A$ in $M$ is $C o n-S$-closed in $M$. Then apply (4) with $B=M$, we have (1).

Remark 3.3. It is clear that if a module $M$ has large radical, then Con- $S$ submodules and submodules of $M$ are the same, i.e. $M$ being extending and $M$ being $S$-extending are equivalent. 
In the following we give simple examples of modules $M$ which are $S$-extending modules, while they are not extending:

(1) The $\mathbb{Z}$-module $M=C(p) \oplus \mathbb{Z}$, for a prime number $p$ is $S$-extending (due to $M$ with no $C o n-S$-submodules), while $M$ is not extending (due to $C(p)$ is not injective as it is shown in [5, Corollary 2]).

(2) A more general than the example in (1), is that the $\mathbb{Z}$-module $M=C\left(p^{n}\right) \oplus$ $\mathbb{Z}$, for a prime number $p, n \in \mathbb{Z}^{+}$is $S$-extending (due to any Con-S-closed submodule $A$ of $M$ must contain $C\left(p^{n}\right)$, and hence either $A=C\left(p^{n}\right)$, or $A=M)$, while $M$ is not extending, for the same reason as in the example in (1).

In the following we show that the $S$-extending condition on a module is inherited by a direct summand.

Lemma 3.4. Let $M$ be a module, and $N$ be a direct summand of $M$. If $M$ is $S$-extending, then so is $N$.

Proof. Let $A$ be a $C o n-S$-closed submodule of $N$. It is clear that $A$ is a $C o n-S$ closed submodule of $M$. As $M$ is an $S$-extending module, it follows that $A$ is a direct summand of $M$ and hence of $N$.

In the following we show that isomorphic copy of an $S$-extending module is $S$-extending.

Lemma 3.5. Let $M$ be an $S$-extending module, and let $f: M \rightarrow N$ be an isomorphism. Then $N$ is an $S$-extending module.

Proof. Let $C$ be a $C o n-S$-closed submodule of $N$. It is easy to show that $f^{-1}(C)$ is a $C$ on- $S$-closed submodule of $M$, and hence $M=f^{-1}(C) \oplus M_{1}$. Thus $N=$ $C \oplus f\left(M_{1}\right)$.

Proposition 3.6. Let $M$ be an $S$-extending module, with $0 \neq \operatorname{Rad}(M)$ not large in $M$. Then $M$ has a decomposition $M=M_{1} \oplus M_{2}$, where $\operatorname{Rad}\left(M_{2}\right)=0$ and $M_{1}$ is extending and $M_{2}$-superfluous-injective.

Proof. Let $M_{1}$ be a maximal large extension of $\operatorname{Rad}(M)$ in $M$. As $M$ is an $S$ extending module, we have that $M=M_{1} \oplus M_{2}$, where $\operatorname{Rad}\left(M_{2}\right)=0$, and hence, by Remark 2.5, $M_{1}$ is $M_{2}$-superfluous-injective. It is clear that $\operatorname{Rad}\left(M_{1}\right)=\operatorname{Rad}(M) \unlhd$ $M_{1}$. By Lemma 3.4, $M_{1}$ is $S$-extending. By Remark 3.3, $M_{1}$ is extending.

Lemma 3.7. ([7, Theorem 4]) $A$ ring $R$ is right noetherian if and only if every extending right $R$-module is a direct sum of uniform submodules. 
Theorem 3.8. Let $M$ be a module over a right noetherian ring $R$, and let $0 \neq \operatorname{Rad}(M)$ not large in $M$. If $M$ is $S$-extending, then $M=M_{1} \oplus M_{2}$, where $\operatorname{Rad}\left(M_{2}\right)=0$; and $M_{1}$ is extending and a direct sum of uniform submodules.

Proof. The proof follows from Proposition 3.6 and Lemma 3.7.

\section{Relative superfluous injective and $S$-extending modules}

In this section we study direct sums of $S$-extending modules which are, in pairs, relatively superfluous injective, and give the necessary and sufficient conditions for such direct sums to be $S$-extending.

Lemma 4.1. Let $M=A+B, A, B$ and $S$ be submodules of a module $M$, and $A \cap B \ll M$. If $S \ll M$, then $A \cap(B+S) \ll M$.

Proof. Let $A \cap(B+S)+C=M$ for some submodule $C$ in $M$. Then by the modular law $A=A \cap M=A \cap(B+S)+(C \cap A)$, hence

$$
M=A+B=A \cap(B+S)+(C \cap A)+B .
$$

Since $A \cap(B+S)+B \subseteq B+S$, then $M \subseteq B+S+(C \cap A) \subseteq M$, hence $M=B+S+(C \cap A)$. Since $S \ll M$, then $M=B+(C \cap A)$. By the modular law, we have that $A=(B \cap A)+(C \cap A)$, and $A \cap(B+S)=(B \cap A)+((C \cap A) \cap(B+S))$. It follows that

$$
M=A \cap(B+S)+C=(B \cap A)+((C \cap A) \cap(B+S))+C=(B \cap A)+C .
$$

Since $A \cap B \ll M$, then $C=M$. Therefore $A \cap(B+S) \ll M$.

Lemma 4.2. Let $M=A \oplus B$ be a module where $A$ has $\left(S_{*}\right)$. Then the following are equivalent:

(1) $B$ is A-superfluous-injective, and $A$ is $S$-extending.

(2) If $C$ is a Con-S-closed submodule of $M$, with $C \cap B=0$, then $M=$ $C \oplus A^{\prime} \oplus B$, for some $A^{\prime} \leq A$.

Proof. (1) $\Rightarrow(2)$ : Let $C$ be a $C o n-S$-closed submodule of $M$, with $C \cap B=0$. Let $0 \neq K \leq C$, with $K \ll M$. By Lemma 4.1 we have that $(K \oplus B) \cap A \ll A$. Since $0 \neq(K \oplus B) \cap A \leq(C \oplus B) \cap A$, it follows that $(C \oplus B) \cap A$ is a $C o n$ - $S$-submodule of $A$. Since $A$ is $S$-extending, then $A=A_{1} \oplus A^{\prime}$ such that $(C \oplus B) \cap A \unlhd A_{1}$. Hence $[(C \oplus B) \cap A] \oplus B \unlhd A_{1} \oplus B$. As $\left.C \oplus B=(C \oplus B) \cap(A \oplus B)=[(C \oplus B) \cap A)\right] \oplus B$, we have that $(C \oplus B) \unlhd A_{1} \oplus B$, and that $C$ is closed in $A_{1} \oplus B$. Thus, by Remark 2.12, it follows that $C$ is a $C o n-S$-complement of $B$ in $A_{1} \oplus B$. Since $B$ 
is $A$-superfluous-injective, it follows, by Proposition 2.6 , that $B$ is $A_{1}$-superfluousinjective. Then, by Theorem 2.16, we have that $A_{1} \oplus B=C \oplus B$. Therefore $M=A \oplus B=A_{1} \oplus A^{\prime} \oplus B=A^{\prime} \oplus C \oplus B$.

$(2) \Rightarrow(1)$ : First we show that $A$ is $S$-extending. To this end let $C$ be a $C o n-S$-closed submodule of $A$. As $A$ is a direct summand of $M$, it follows that $C$ is a $C o n-S$-closed submodule of $M$, with $C \cap B=0$. By (2), $C$ is a direct summand of $M$, and hence is a direct summand of $A$. Now we show that $B$ is $A$-superfluous-injective. Let $D$ be a $C o n-S$-complement of $B$ in $M$, hence, by Remark 2.12, $D$ is Con-S-closed submodule of $M$; with $D \cap B=0$. By (2), we have that $M=A^{\prime} \oplus D \oplus B$, for some submodule $A^{\prime}$ of $A$. As $D$ is a $C o n-S$-complement of $B$ in $M$, and $\left(D \oplus A^{\prime}\right) \cap B=0$, we have that $D=D \oplus A^{\prime}$; i.e. $M=D \oplus B$. Therefore, by Theorem $2.16, B$ is $A$-superfluous-injective.

Theorem 4.3. Let $M=M_{1} \oplus M_{2}$ be a module where $M_{1}$ has $\left(S_{*}\right)$. Then the following are equivalent:

(1) $M_{i}$ is $M_{j}$-superfluous-injective $(i \neq j=1,2)$, and $M_{i}$ is $S$-extending $(i=1,2)$.

(2) If $C$ is a Con-S-closed submodule of $M$, with $C \cap M_{i}=0(i=1$ or 2$)$, then $M=C \oplus M_{j}^{\prime} \oplus M_{i}$, for some $M_{j}^{\prime} \leq M_{j}, j \neq i$.

Proof. The proof follows from Lemma 4.2.

Corollary 4.4. Let $M=M_{1} \oplus M_{2}$ be a module, with $M_{i}(i=1,2)$ uniform. If $M_{i}$ is $M_{j}$-superfluous-injective $(i \neq j=1,2)$, then $M$ is $S$-extending.

Proof. The proof follows from Theorem 4.3, and from the fact that every Con-Sclosed submodule $C$ of $M$, different from $M$, is uniform; and hence $C \cap M_{i}=0$ for some $i=1,2$.

Remark 4.5. As an application of Corollary 4.4, we have the following examples:

(1) Direct sums of two $S$-extending modules need not, in general, be $S$-extending modules; for the $\mathbb{Z}$-module $C(p) \oplus C\left(p^{3}\right)$, for a prime number $p$, is not $S$ extending (due to $C(p)$ not a $C\left(p^{3}\right)$-superfluous-injective).

(2) The $\mathbb{Z}$-module $C\left(p^{n}\right) \oplus \mathbb{Z}$, where $n \in \mathbb{Z}^{+}$and $p$ is a prime number, is an $S$ extending $\mathbb{Z}$-module (due to $C\left(p^{n}\right)$ and $\mathbb{Z}$ relatively superfluous-injective), which is not extending as we have mentioned and given the reason before.

Definition 4.6. Let $M=M_{1} \oplus M_{2}$ be a module. $M$ is said to satisfy the superfluous closed intersection property (for short $M$ satisfy the $S I C$ property), if for any 
$0 \neq K \ll M$, with $K \cap M_{i}=0$, for some $i=1,2$, then $C \cap M_{i}=0$ for each proper closed submodule $C$ of $M$ containing $K$.

It is clear that if $M$ and $K$, the same as in Definition 4.6, with $K \cap M_{i}=0$, then $C \cap M_{i}=0$ for each maximal large extension $C$ of $K$ in $M$.

Proposition 4.7. Let $M=M_{1} \oplus M_{2}$ be a module, which satisfies the SIC property. Then the following are equivalent:

(1) $M$ is an $S$-extending module.

(2) Every Con-S-closed submodule $C$ of $M$, with $C \cap M_{i}=0$, for some $i=$ 1 or 2 , is a direct summand of $M$.

Proof. $(1) \Rightarrow(2)$ : It is clear.

$(2) \Rightarrow(1)$ : It is clear that each $C o n-S$-closed submodule $C$ in $M_{i}$ is a $C o n-S$ closed submodule of $M$, with $C \cap M_{j}=0, i \neq j$. Hence $M_{i}(i=1,2)$ is an $S$ extending module. Now let $C$ be a $C o n-S$-closed submodule of $M$, with $C \cap M_{i} \neq 0$, $i=1,2$. As $C$ is a $C o n-S$-submodule of $M$, there exists a submodule $K$ in $C$ with $0 \neq K \ll M$. Since $M$ satisfies the $S I C$ Property, and since $C \cap M_{1} \neq 0$, we have that $0 \neq K \cap M_{1} \leq C \cap M_{1}$ is a $C o n-S$-submodule of $M$. Let $C^{\prime}$ be a maximal large extension of $C \cap M_{1}$ in $C$. It is clear that $C^{\prime}$ is a $C o n-S$-closed submodule of $M$, with $C^{\prime} \cap M_{2}=0$. Hence, by (2), $M=C^{\prime} \oplus H$, for some submodule $H$ of $M$. It follows that $C=C^{\prime} \oplus C^{\prime \prime}$, where $C^{\prime \prime}=C \cap H$. Let $\pi: M \rightarrow H$ be the projection onto $H$. Again, since $M$ satisfies the $S I C$ Property, we have that $0 \neq K \cap M_{2} \ll M$. As $\left(K \cap M_{2}\right) \cap C^{\prime}=0$, we have that $0 \neq \pi\left(K \cap M_{2}\right) \leq C^{\prime \prime}$, with $\pi\left(K \cap M_{2}\right) \ll M$. Hence $C^{\prime \prime}$ is a $C$ on-S-closed submodule of $M$, with $C^{\prime \prime} \cap M_{1}=0$. Thus, by (2), $C^{\prime \prime}$ is a direct summand of $M$, and therefore $C$ is a direct summand of $M$.

Lemma 4.8. Let $M=M_{1} \oplus M_{2}$ be a module, with $0 \neq \operatorname{Rad}(C) \unlhd C$, for every proper Con-S-closed submodule $C$ of $M$, with $C \cap M_{i} \neq 0, i=1$, 2. Then the following are equivalent:

(1) $M$ is an $S$-extending module.

(2) Every Con-S-closed submodule $C$ of $M$, with $C \cap M_{i}=0$, for some $i=1$ or 2 , is a direct summand of $M$.

Proof. $(1) \Rightarrow(2)$ : It is clear.

$(2) \Rightarrow(1)$ : It is clear that each $C o n-S$-closed submodule $C$ in $M_{i}$ is a $C o n-S$-closed submodule of $M$, with $C \cap M_{j}=0, i \neq j$. Hence $M_{i}(i=1,2)$ is an $S$-extending module. Now let $C$ be a proper $C o n$ - $S$-closed submodule of $M$, with $C \cap M_{i} \neq 0$, 
$(i=1,2)$, and with $0 \neq \operatorname{Rad}(C) \unlhd C$. It follows that $C \cap M_{1}$ is a $C o n-S$-submodule of $M$; and hence $M=C^{\prime} \oplus L$, where $C^{\prime}$ is a maximal large extension of $C \cap M_{1}$ in $C$, and $L$ is a submodule of $M$. It follows that $C=C^{\prime} \oplus(L \cap C)$. If $L \cap C=0$, then we have done. On the other hand, if $C \cap L \neq 0$, then $C \cap L$ is a $C o n-S$-closed submodule of $M$, with $(C \cap L) \cap M_{1}=0$ (due to $\operatorname{Rad}(C) \unlhd C$ ). Again, by (2), $C \cap L$ is a direct summand of $M$, and therefore $C$ is a direct summand of $M$.

Theorem 4.9. Let $M=M_{1} \oplus M_{2}$ be a module, with $0 \neq \operatorname{Rad}(C) \unlhd C$, for every proper Con-S-closed submodule $C$ of $M$, with $C \cap M_{i} \neq 0,(i=1,2)$. Let $M_{i}$ be $M_{j}$-superfluous-injective, $(i \neq j=1,2)$. Then the following are equivalent:

(1) $M$ is an $S$-extending module.

(2) $M_{1}$ and $M_{2}$ both are $S$-extending modules.

Proof. $(1) \Rightarrow(2)$ : Follows from Lemma 3.4.

$(2) \Rightarrow(1)$ : By Lemma 4.8, it is enough to show that every $C o n$ - $S$-closed submodule $C$ of $M$, with $C \cap M_{i}=0$ for some $i=1$ or 2 , is a direct summand of $M$. To this end, let $C$ be a $C o n-S$-closed submodule of $M$, with $C \cap M_{1}=0$. Since $M_{1}$ is $M_{2}$-superfluous-injective and $M_{2}$ is $S$-extending, we have, by Lemma 4.2 , that $M=C \oplus M_{2}^{\prime} \oplus M_{1}$, for some $M_{2}^{\prime} \leq M_{2}$.

Theorem 4.10. Let $M=M_{1} \oplus M_{2}$ be a module, which satisfies the SIC property. Let $M_{i}$ be $M_{j}$-superfluous-injective, $(i \neq j=1,2)$. Then the following are equivalent:

(1) $M$ is an $S$-extending module.

(2) $M_{1}$ and $M_{2}$ both are $S$-extending modules.

Proof. The proof follows from Lemma 3.4, Proposition 4.7 and Lemma 4.2.

Corollary 4.11. Let $M=\oplus_{i=1}^{n} M_{i}$ be a module, $0 \neq \operatorname{Rad}(C) \unlhd C$, for every proper Con-S-closed submodule $C$ of $M$, with $C \cap M_{i} \neq 0,(i=1,2, \ldots, n)$. Let $M_{i}$ be $M_{j}$-superfluous-injective, $(i \neq j=1,2, \ldots, n)$. Then the following are equivalent:

(1) $M$ is an $S$-extending module.

(2) $M_{i}$ is $S$-extending modules, for each $i=1,2, \ldots, n$.

Proof. The proof follows by Theorem 4.9, and induction on $n$.

Acknowledgement. The authors would like to thank the referee for the valuable suggestions and comments. 


\section{References}

[1] F. W. Anderson and K. R. Fuller, Rings and Categories of Modules, Second edition, Graduate Texts in Mathematics, 13, Springer-Verlag, New York, 1992.

[2] W. D. Burgess and R. Raphael, On modules with the absolute direct summand property, Ring Theory (Granville, OH, 1992), World Sci. Publ., River Edge, NJ, (1993), 137-148.

[3] H. Q. Dinh, A note on pseudo injective modules, Comm. Algebra, 33(2) (2005), 361-369.

[4] N. V. Dung, D. V. Huynh, P. F. Smith and R. Wisbaur, Extending Modules, with the collaboration of John Clark and N. Vanaja, Pitman Research Notes in Mathematics Series, 313, Longman Scientific \& Technical, Harlow; copublished in the United States with John Wiley \& Sons, Inc., New York, 1994.

[5] M. A. Kamal and B. J. Müller, Extending modules over commutative domains, Osaka J. Math., 25(3) (1988), 531-538.

[6] E. Mermut, C. Santa-Clara and P. F. Smith, Injectivity relative to closed submodules, J. Algebra, 321(2) (2009), 548-557.

[7] M. Okado, On the decomposition of extending modules, Math. Japon., 29(6) (1984), 939-941.

[8] M. F. Yousif and Y. Zhou, FP-injective, simple-injective, and quasi-Frobenius rings, Comm. Algebra, 32(6) (2004), 2273-2285.

Manar E. Tabarak (Corresponding Author) and Salah El Din S. Hussien

Department of Mathematics

Faculty of Sciences

Ain Shams University

Cairo, Egypt

e-mails: manarelbadry@sci.asu.edu.eg (M. E. Tabarak)

mynsalah@hotmail.com (S. E. D. S. Hussien)

M. A. Kamal and M. H. Elbaroudy

Department of Mathematics

Faculty of Education

Ain Shams University

Roxy, Cairo, Egypt

e-mails: mahmoudkamaleldeen@edu.asu.edu.eg (M. A. Kamal)

mahaelbarode@edu.asu.edu.eg (M. H. Elbaroudy) 